\title{
$\beta$-Elemene Reverses Chemoresistance of Breast Cancer via Regulating MDR-Related MicroRNA Expression
}

\author{
Jun Zhang ${ }^{a, c, g}$ He da Zhang ${ }^{a, b}$ Lin Chen ${ }^{a, b}$ Da Wei Sun ${ }^{a, e}$ Chang fei Mao ${ }^{a, d}$ \\ Wei Chen ${ }^{a, b}$ Jian Zhong Wu ${ }^{f}$ Shan liang Zhong ${ }^{f}$ Jian Hua Zhao ${ }^{f}$ Jin Hai Tanga \\ aDepartment of General Surgery, Nanjing Medical University Affiliated Cancer Hospital, Cancer Institute \\ of Jiangsu Province, Nanjing, b'Graduate School, Xuzhou Medical College, Xuzhou, 'Anhui University of \\ Chinese Medicine, Anhui, Feihe, 'Nanjing Medical University, Nanjing, eNanjing University of Chinese \\ Medicine, Nanjing, 'Department of Center of Clinical Laboratory Science, Nanjing Medical University \\ Affiliated Cancer Hospital, Cancer Institute of Jiangsu Province, Nanjing, ${ }^{9}$ Department of postDoctoral \\ working station, Jinling Hospital Affiliated to Medical College of Nanjing University, Nanjing, Jiangsu, \\ China
}

\section{Key Words}

Breast cancer $\bullet$ Chemoresistance $\bullet \beta$-elemene $\cdot$ miRNA

\begin{abstract}
Background: Multidrug resistance (MDR) directly contributes to the clinical failure of chemotherapy in breast cancer (BCA). $\beta$-elemene is a natural antitumor drug from plants. We previously confirmed that MDR could be reversed by $\beta$-elemene. In this study, we intended to investigate the reversal effect of $\beta$-elemene on MDR in human BCA adriacin (Adr) resistant MCF-7 cells (MCF-7/Adr) and docetaxel (Doc) - resistant MCF-7 cells (MCF-7/Doc) through the gene regulatory network. Methods: MTT-cytotoxic, miRNA microarray, Real-time quantitative PCR, Dual Luciferase Activity Assay ,Western blot analysis were performed to investigate the impact of $\beta$-elemene on chemo-resistant BCA cell suvival, and its impact on the expression of chemo-resistance specific miRNA and the downstream target genes PTEN and Pgp. Results: Compared with the miRNAs expression profiles of MCF-7/Adr and MCF7/Doc cell lines from our previous studies, there were 322 differentially expressed miRNAs in MCF-7/Adr and MCF-7/Doc breast cancer cells with $\beta$-elemene intervention (50 $\mu \mathrm{M} / \mathrm{L})$ for $30 \mathrm{~h}$, and 6 miRNAs were significantly up-regulated and 12 miRNAs were significantly downregulated in both MCF-7/Adr and MCF-7/Doc. We have testified that 5 miRNA is related to MDR before, in this study, the expression of miR-34a, miR-222, miR-452 and miR-29a can lead to changes of the characteristics of chemo-resistant MCF-7/Adr and MCF-7/Doc. The PTEN expression under intervention of $\beta$-elemene was significantly increased and Pgp expression under $\beta$-elemene intervention was significantly decreased in both cell lines. Conclusions: $\beta$-elemene could influence MDR related miRNA expression and subsequently regulate the

J. Zhang and H.-d. Zhang contributed equally to this work.


expression of the target genes PTEN and Pgp, which may lead to reduction of the viability of the chemo-resistant breast cancer cells.

Copyright $(2014$ S. Karger AG, Basel

\section{Introduction}

Breast cancer is the most common cancer in women and a major cause of cancer mortality. Current treatment strategies combine surgery with adjuvant therapy, but chemo-resistance and toxicity are the leading causes that limit the success of treatment towards the aggressive breast cancer cases. Elemene (1-methyl-1-vinyl-2, 4-Diisopropenyl-cyclohexane) isolated from the Chinese medicinal herb Rhizoma Zedoariae, is a novel noncytotoxic anticancer drug $[1,2]$. The extract of elemene is a mixture of $\beta, \delta$ and $\gamma$-elemene, with $\beta$-elemene as the main component, accounting for $60-72 \%$ of the three isoforms. Previous studies have provided abundant evidence to reveal that $\beta$-elemene might be an effective MDR reversing agent in cancer chemotherapy and mainly via inhibition of the transport activity of Pgp [3, 4]. However the underline mechanism has not been fully elucidated.

MicroRNAs (miRNAs) are a new class of small, nonprotein-encoding RNAs that range in size from 19 to 25 nucleotides (nt) and have important roles in a variety of biologic processes [5-7], and also have a very important role in tumorigenesis, development, cellular migration, apoptosis, signal transduction and carcinogenesis. Recently, accumulating evidence is revealing an important role of miRNAs in anticancer drug resistance and miRNAs expression profiling can be correlated with the development of anticancer drug resistance, such as miR21, miR-22, miR-155, miR-181a, miR-34a, miR-222, etc. There are several mechanisms have been shown to be targeted by miRNAs in drug-resistant breast cancer such as DNA repair [8-11].

There are data which suggest that $90 \%$ of patients who died of cancer are connected with chemoresistance. Adriamycin (Adr) and Docetaxel (Doc) are two of the most common chemotherapy drugs. One important reason for the failure of chemotherapy is primary or acquired resistance. Multi-drug resistance (MDR) means that tumor cells with long-term exposure to a single chemotherapy drug may become resistant to a wide range of different structures -- different targets of anticancer drugs. The combined treatment of $\beta$-elemene with ADR or DOC at non-effect dosage lead to higher inhibition efficiencies and increased cell death rate, implying the excellent ability of $\beta$-elemene in reversing the multi-drug resistance of MCF-7 cells. We can currently propose that $\beta$-elemene with anti-cancer agents may be effective in multi-drug resistant breast cancer by down-regulating MDR1 proteins [12].

From recent studies, $\beta$-elemene has revealed to have an apparent synergistic effect over chemotherapeutic agents in cancer cells. However, there has been no report to demonstrate the mechanisms of $\beta$-elemene to reverse MDR in breast cancer from miRNA levels. Our team conducted the profiling of miRNAs expression in MCF-7/Adr and MCF-7/Doc cell lines, we are the first to report the findings and testify that 5 miRNA is related to MDR. We also revealed that $\beta$-elemene modulated the expression of MDR-related miRNAs and proteins, which may contribute to reversing the BCA chemo-resistance. We propose a logical hypothesis: $\beta$-elemene could mediate the MDR specific miRNA, which could then regulate the downstream target and corresponding target genes through the gene regulatory network to interrupt the development process of drug-resistance in cancer cells, hence to improve the treatment efficacy.

\section{Materials and Methods}

\section{Cell culture}

Human breast cancer cell line MCF-7 was purchased from ATCC (Rockville, MD). The resistant sublines, selected at 100nm docetaxel (MCF-7/Doc) or at 500nm Adriamycin (MCF-7/Adr), were successfully 
established from human breast cancer parental cell line MCF-7 by exposing MCF-7 to gradually increasing concentrations of Doc or Adr in vitro in our laboratory. The IC50 (inhibitory concentration to produce $50 \%$ cell death) values of Adr were 403.56 and $0.66 \mu \mathrm{M}$ for MCF-7/Adr and MCF-7/S cells, respectively. The IC50 values of Doc in MCF-7/Doc and MCF-7/S cells were 68.31 and 3.08 $\mu \mathrm{M}$, respectively. All cell lines were cultured in DMEM high glucose (HyClone), supplemented with $10 \%$ fetal bovine serum (Gibco) in a humidified atmosphere containing $5 \% \mathrm{CO}_{2}$ at $37^{\circ} \mathrm{C}$.

\section{MTT-cytotoxic}

Cells were seeded into 96 -well plates $\left(6 \times 10^{3}\right.$ cells/well $)$, treated with different concentrations of $\beta$-elemene and incubated for 48 hours. Then $20 \mu \mathrm{l}$ of MTT solution $(5 \mathrm{mg} / \mathrm{ml})$ was added to each well and the cells were maintained in a humidified atmosphere for $3-4$ hours at $37^{\circ} \mathrm{C}$. The MTT-containing medium was removed and 150lL of DMSO (AMRESCO, America) was added to each well; each experiment was performed in quadruplicate. The absorbance was measured at 570nm using CliniBio128 (ASYS-Hitech, Austria).

\section{Total RNA extraction and miRNA microarray}

Total RNA including miRNAs was extracted using MirVana miRNA Isolation Kit (Ambion, AM1560). The concentration and quality of the RNA were measured by the UV absorbance at 260 and $280 \mathrm{~nm}$ (260/280 nm) on Nanodrop 2000 spectrophotometry (Thermo Scientific) and by formaldehyde denaturing gel electrophoresis. The RNA was labeled using the FlashTag RNA Labeling Kit (Genishere), according to Affymetrix manufacturer's recommendations. First, poly(A) tailing was carried out at $37{ }^{\circ} \mathrm{C}$ for 15 min in a volume of $15 \mu \mathrm{l}$ reaction mix, which contains $1 \times$ Reaction Buffer, $1.5 \mu \mathrm{l} 25 \mathrm{mM} \mathrm{MnCl2,} 1 \mu \mathrm{l}$ 1:500 diluted ATP Mix and $1 \mu$ PAP enzyme. Second, FlashTag Ligation was performed at room temperature for 30 min by adding $4 \mu \mathrm{l}$ of $5 \times$ FlashTag Ligation Mix Biotin and $2 \mu \mathrm{l}$ T4 DNA Ligase into the $15 \mu \mathrm{l}$ of reaction mix. 2.5 $\mu \mathrm{l}$ of Stop Solution was added to stop the reaction. Hybridization and washing were performed using the Affymetrix Fluidics Station 450 and Hybridization Oven 640 under standard conditions. Image processing was conducted using the Affymetrix GeneArray 3000 scanner. The Affymetrix GeneChip miRNA 2.0 Array contains 15,644 probe sets including 1105 human mature miRNAs. The raw data was treated using miRNA QC tool software (Affymetrix). The data output was received in Excel spreadsheets containing the normalized micro-RNA expression profiles. Differentially expressed miRNAs werefiltered to exclude those changes less than 2.0-fold compared with MCF-7/S.

\section{Real-time quantitative PCR}

Total RNA was extracted using TRIzol ${ }^{\circledR}$ Reagent (Invitrogen, Carlsbad, CA); afterwards a reverse transcription was done using TaqMan ${ }^{\circledR}$ MicroRNA Reverse Transcription Kit (Applied Biosystems, Foster City, CA); mature miRNA was spotted using TaqMan ${ }^{\circledR}$ MicroRNA Assay (Applied Biosystems, Foster City, $\mathrm{CA}$ ); all procedures were done according to manufacturer's instructions. Relative expression levers were calculated using the $\Delta \Delta \mathrm{Ct}$ method, normalized with endogenous control and was presented along with negative control. Clustal X software was used to analyze measured miRNA sequences; the sequences were similarity not high in a reverse transcription system. All reverse transcriptions and PCR assays were presented in triplicate.

\section{Dual Luciferase Activity Assay}

In order to facilitate observation, a recombinant lentiviral vector stable expression of green fluorescent protein was used in breast cancer cell lines. MCF-7/Doc and MCF-7 cells in logarithmic growth phase were seeded in 24 -well plates $\left(3 \times 10^{4}\right.$ cells/well) after digestion until cell fusion becomes $50 \%$ to $60 \%$; which was carried out in accordance with reagent instruction lentivirus infections. After 72 hours, the collected fluorescence was stronger in each well, which resurfaced after digestion was covered with $50 \%$ to $60 \%$; added $2 \mu \mathrm{g} / \mathrm{ml}$ puromycin to screen. After one week, $1 \mu \mathrm{g} / \mathrm{ml}$ puromycin was added to maintain the pressure; three generations were continued to be cultured to observe the expression of the green fluorescence. Then, the green fluorescence MCF-7 cells, MCF-7/Adr cells and MCF-7/Doc cells were inoculated for 24 hours in equal amounts with $\beta$-elemene intervention; then afterwards intervened with Doc and Adr for 24 hours. Luciferase activities were measured using a Dual Luciferase Reporter Assay System (Promega, USA) according to the manufacturer's instruction and the renilla luciferase activity was normalized. 
Fig. 1. $\beta$-elemene treatment restrains the viability of human breast cancer cells. We utilized different $\beta$-elemene concentrations to detect the activitiesy of MCF-7(Fig. 1A), MCF-7/Doc (Fig. 1B) and MCF-7/Adr (Fig. 1C) cells by MTT-cytotoxic. The cell lines were treated with DMSO (control) or different concentrations of $\beta$-elemene $(10,30$, 50 , and $100 \mu \mathrm{M} / \mathrm{L}$ ) for $30 \mathrm{~h}$. All data corresponds to the mean $\pm S D$ of three independent experiments. Significantly different compared from with control by one-way ANOVA, ${ }^{* *} \mathrm{p}<0.01,20$ and $25 \mu \mathrm{M} / \mathrm{L}$ vs $0-15 \mu \mathrm{M} / \mathrm{L}$ (Fig. $1 \mathrm{~A}$ ), $100 \mu \mathrm{M} / \mathrm{L}$ vs $0-50 \mu \mathrm{M} / \mathrm{L}$ (Fig. 1B), 50 and $100 \mu \mathrm{M} / \mathrm{L}$ vs $0-30$ $\mu \mathrm{M} / \mathrm{L}$ (Fig. 1C).

\section{Western blot}

Total protein was extracted and lysed in the RIPA buffer (Beyotime, Jiangsu, China). Equal amounts of proteins were separated by $10 \%$ SDS-PAGE and transferred to the polyvinylidene difluoride membranes (Sigma, Germany). After blocking with 5\% skim milk, the membranes were incubated with primary antibodies against human PTEN and Pgp (1:100, Abcam, America) overnight at $4^{\circ} \mathrm{C}$; after washing with TBS, the horseradish peroxidase-conjugated secondary antibody (Kangwei Ltd., Beijing, China) was further incubated; the protein band was visualized by Chemiluminescence with pierce ECL kits (Millipore, Billerica, MA). $\beta$-actin (1:4000, Bioworld, MN) was used as an internal load to normalize the expression patterns of each sample. Three separate experiments were performed to show the protein expression.

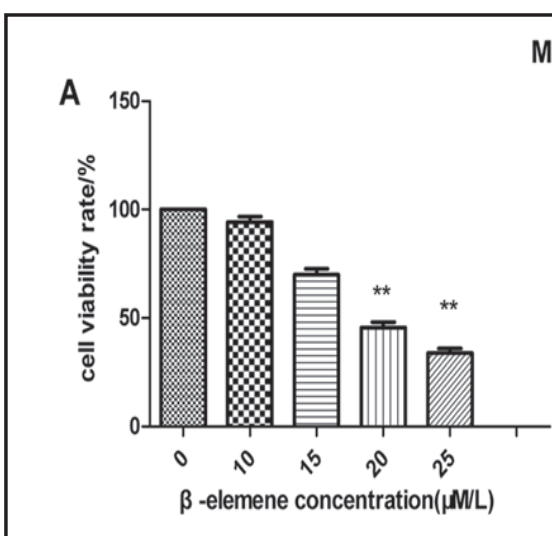

B

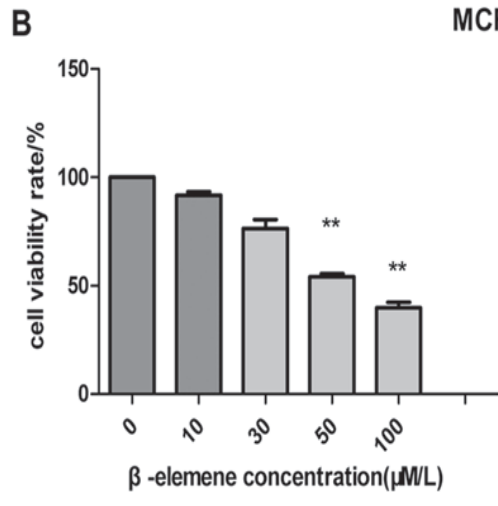

C

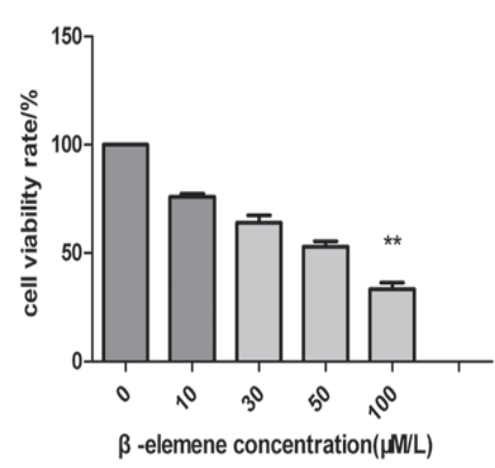

\section{Statistical analysis}

All experiments were performed in triplicate and a representative data was shown from three separate experiments. A statistical analysis was performed using a t-test or One-way ANOVA and Spearman rank test with a SPSS 16.0 statistic. All experiments were performed in triplicate; $p<0.05$ was considered statistically significant.

\section{Results}

The viability of chemo-resistant breast cancer cell lines after intervention with different concentrations of $\beta$-elemene

Some studies show that $\beta$-elemene treatment effect human breast cancer cells. In this study, we utilized different $\beta$-elemene concentrations and exposure times to determine its impact on cell proliferation. As shown in Fig. 1, the viability of MCF-7/Doc (Fig. 1A), MCF- 
Table 1. Significantly changed miRNAs by miRNA microarray of the expression profile in MCF-7/Doc and MCF-7/Adr cells with $\beta$-elemene intervention $(50 \mu \mathrm{M} / \mathrm{L})$ for $30 \mathrm{~h}$ compared with the expression of miRNAs in MCF-7/Doc and MCF-7/Adr cells we tested before and confirmed targets or pathway, (Criteria differences for Ratio > 2.0, compared to MCF-7)

\begin{tabular}{|c|c|c|c|c|c|}
\hline $\begin{array}{l}\text { Significantly } \\
\text { up-regulation }\end{array}$ & $\mathrm{FC}$ & $\begin{array}{l}\text { Confirmed target } \\
\text { or pathway }\end{array}$ & $\begin{array}{l}\text { Significantly } \\
\text { down-regulation }\end{array}$ & FC & $\begin{array}{l}\text { Confirmed targets or } \\
\text { pathway }\end{array}$ \\
\hline miR-320d & 2.56 & & miR-155 & 2.75 & FOXO3 RHOA \\
\hline miR-34a & 6.83 & NOTCH1 BIRC3 & miR-145 & 2.21 & ESR1 IGFR IRS1 \\
\hline miR-4284 & 2.29 & & $\operatorname{miR}-424$ & 3.24 & \\
\hline miR-500 & 3.05 & & $\operatorname{miR}-455$ & 3.68 & \\
\hline $\operatorname{miR}-193 b$ & 2.99 & & miR-503 & 2.21 & \\
\hline \multirow[t]{7}{*}{$\mathrm{miR}-200 \mathrm{c}$} & 3.56 & PGP & $\operatorname{miR}-29 a$ & 8.32 & PTEN \\
\hline & & & miR-125b & 5.77 & BCL-2 \\
\hline & & & miR-222 & 2.63 & PTEN P27 BMF \\
\hline & & & miR-767-5p & 3.39 & \\
\hline & & & miR-152 & 2.17 & DNMT1 \\
\hline & & & miR-452 & 7.91 & TCF4, LEF1, Bmi-1 \\
\hline & & & MiR-181a & 6.63 & BCRP/ABCG2 \\
\hline
\end{tabular}

7/Doc (Fig. 1B) and MCF-7/Adr (Fig. 1C) cells was decreased with the increased levels of $\beta$-elemene concentration. MCF-7/Adr cells with $\beta$-elemene $(100 \mu \mathrm{M} / \mathrm{L})$ intervention and MCF-7/Doc with $\beta$-elemene $(50 \mu \mathrm{M} / \mathrm{L})$ intervention displayed dramatic decrease of cell viability with statistical significance; In particular, MCF-7/Doc and MCF-7/Adr cells exhibited a relatively high sensitivity to $\beta$-elemene. These suggest that $\beta$-elemene has a strong antiproliferative activity in chemo-resistant MCF-7 cell lines. As shown in Fig. 2, The three cell lines with $\beta$-elemene ( $50 \mu \mathrm{M} / \mathrm{L})$ treatment for 10 or 30 hours showed significant decrease of cell viability. Hence, these suggest that $\beta$-elemene treatment reduces the viability of chemoresistant breast cancer cells in a dose and time-dependent manner.

Expression profile of miRNAs in MCF-7/Doc cells and MCF-7/Adr cells with $\beta$-elemene intervention

In our previous studies, we tested the expression profile of miRNAs in MCF-7/Adr, MCF7/Doc and MCF-7/S cells. Compared with MCF-7/S cell line, there were 183 differentially expressed miRNAs (at least 2.0-fold changes) in MCF-7/Adr and MCF-7/Doc cells. Among the 183 miRNAs, 10 miRNAs were up-regulated, while 26 miRNAs were down-regulated in both MCF-7/Adr and MCF-7/Doc cells [10].

In this study, the expression profile of miRNAs in MCF-7/Doc cells and MCF-7/Adr cells with $\beta$-elemene ( $50 \mu \mathrm{M} / \mathrm{L})$ intervention for $30 \mathrm{~h}$ were evaluated using an Affymetrix GeneChip miRNA 2.0 Array; screened differentially expressed miRNA and validated through real-time quantitative PCR (primer stem-loop RT-PCR method). Compared with MCF-7/Doc and MCF-7/Adr cells without $\beta$-elemene intervention, there were 322 differentially expressed miRNAs among 1,200 miRNAs (criteria differences for Ratio $>2.0$ or $<0.5$, compared to MCF7). Among the 322 miRNAs, 65 miRNAs were correlated with the constant changes of the MDR in two cell lines, 18 miRNAs were up-regulated, and 47 miRNAs were down-regulated in both MCF-7/Adr and MCF-7/Doc. 89 miRNAs were up-regulated and 56 miRNAs were down-regulated in MCF-7/Doc only, 109 miRNAs were up-regulated and 68 miRNAs were down-regulated in MCF-7/Adr only. There were 25 miRNAs up-regulated in MCF-7/Doc but down-regulated in MCF-7/Adr, and 21 miRNAs down-regulated in MCF-7/Doc but upregulated in MCF-7/Adr (Fig. 3).

Among the 322 miRNAs, 6 miRNAs were up-regulated, and 12 miRNAs were downregulated in both MCF-7/Adr and MCF-7/Doc cells significantly (criteria differences for Ratio $>4.0$ or $<0.2$, compared to MCF-7) (Table 1 ). 
Fig. 2. Effect of $\beta$-elemene treatment on viability of MCF-7 cell lines by MTT-cytotoxic. Except MCF-7 cells, other groups were treated with $\beta$-elemene $(50 \mu \mathrm{M} / \mathrm{L})$ during in the corresponding period $(0 \mathrm{~h}, 3 \mathrm{~h}, 10 \mathrm{~h}$, $30 \mathrm{~h})$. All data corresponds to the mean \pm SD of the three independent experiments. Significantly different compared from with control by one-way ANOVA, \#\#** $<0.01$, MCF-7/Doc or MCF-7/Adr vs MCF-7/Doc $+\beta$-elemene.

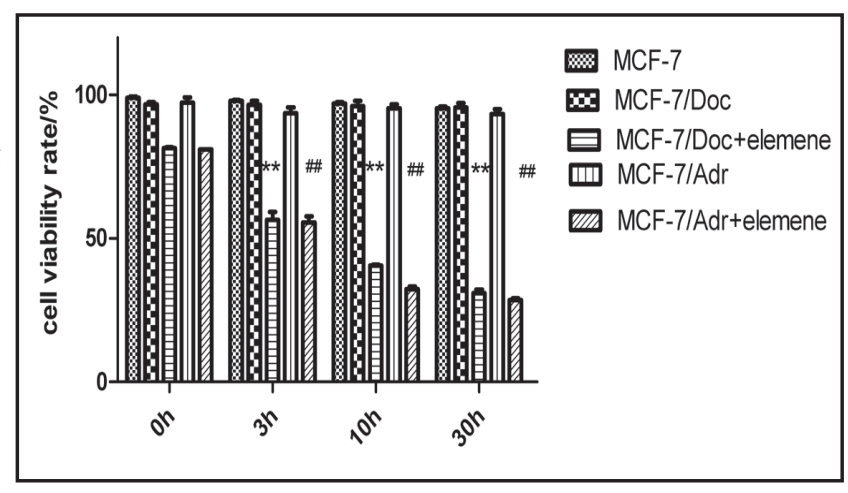

Fig. 3. By miRNA microarray, compared with the miRNA profile of MCF-7/Doc and MCF-7/Adr cells we tested before, it shows the percentage distribution of the 322 differentially expressed miRNAs of expression profile in MCF-7/Doc cells and MCF-7/Adr cells with $\beta$-elemene intervention $(50 \mu \mathrm{M} / \mathrm{L})$ for $30 \mathrm{~h}$.

\section{$\beta$-elemene can reverse chemo-resistance}

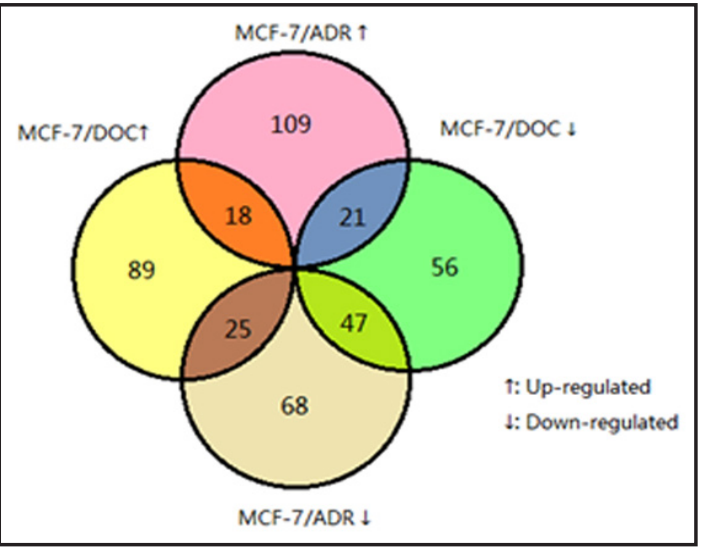

After MCF-7/Doc and MCF-7/Adr cells with $\beta$-elemene intervention $(50 \mu \mathrm{M} / \mathrm{L})$ for $30 \mathrm{~h}$, divided into two groups, the Doc treated and Doc untreated group, and the Adr treated and Adr untreated group. By Doc and Adr, remnants from green fluorescent cells had significant differences between MCF-7/Doc, MCF-7/Adr and MCF-7 cells; proving the existence of MCF7/Doc and MCF-7/Adr drug resistance. However, As seen in Figure 4C, 4D, after treated with $50 \mathrm{~nm}$ Doc or $250 \mathrm{~nm}$ Adr for $30 \mathrm{~h}$, Consistent with a decrease of the residual GFP number, MCF-7/Doc or MCF-7/Adr cells compared to MCF-7/Doc or MCF-7/Adr cells with $\beta$-elemene intervention, we can show that co-culture with $\beta$-elemene with MCF-7/Doc or MCF-7/Adr may significantly promote apoptosis induced by toxic insult. These suggested that MCF-7/ Doc and MCF-7/Adr with $\beta$-elemene intervention, could potentially reverse chemoresistance to recipient cells.

\section{$\beta$-elemene reverses breast cancer cell resistance by mediating related-miRNA}

In previous study, we also testified that 5 miRNA is related to MDR (miR-34a $\downarrow$, miR-

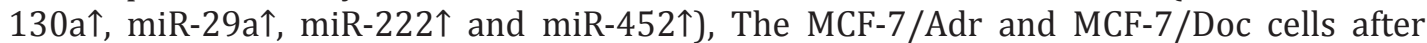
$\beta$-elemene intervention $(50 \mu \mathrm{M} / \mathrm{L})$ for $30 \mathrm{~h}$; and the expression of five drug-specific miRNAs that were compared to MCF-7/Adr and MCF-7/Doc without $\beta$-elemene intervention. After evaluated using an Affymetrix GeneChip miRNA 2.0 Array, significant differences were found before and after $\beta$-elemene intervention. Among the five drug-specific miRNAs in MCF-7/Adr and MCF-7/Doc, four of them have significant reversal changes (miR-34a $\uparrow$, miR-222 $\downarrow$, miR$452 \downarrow$, miR-29a $\downarrow$ ) (Fig. 5). This suggests $\beta$-elemene may modulate the MDR specific miRNA to reverse breast cancer cell chemo-resistance.

$\beta$-elemene treatment alters the expression of PTEN and Pgp protein in breast cancer cells

In order to further verify if $\beta$-elemene can reverse breast cancer cell resistance to chemo-agents, we utilized western blot to detect whether the potential expression changes of PTEN and Pgp. As we know, the expression of the PTEN and PGP has an important role in BCA drug resistance $[13,14]$. 


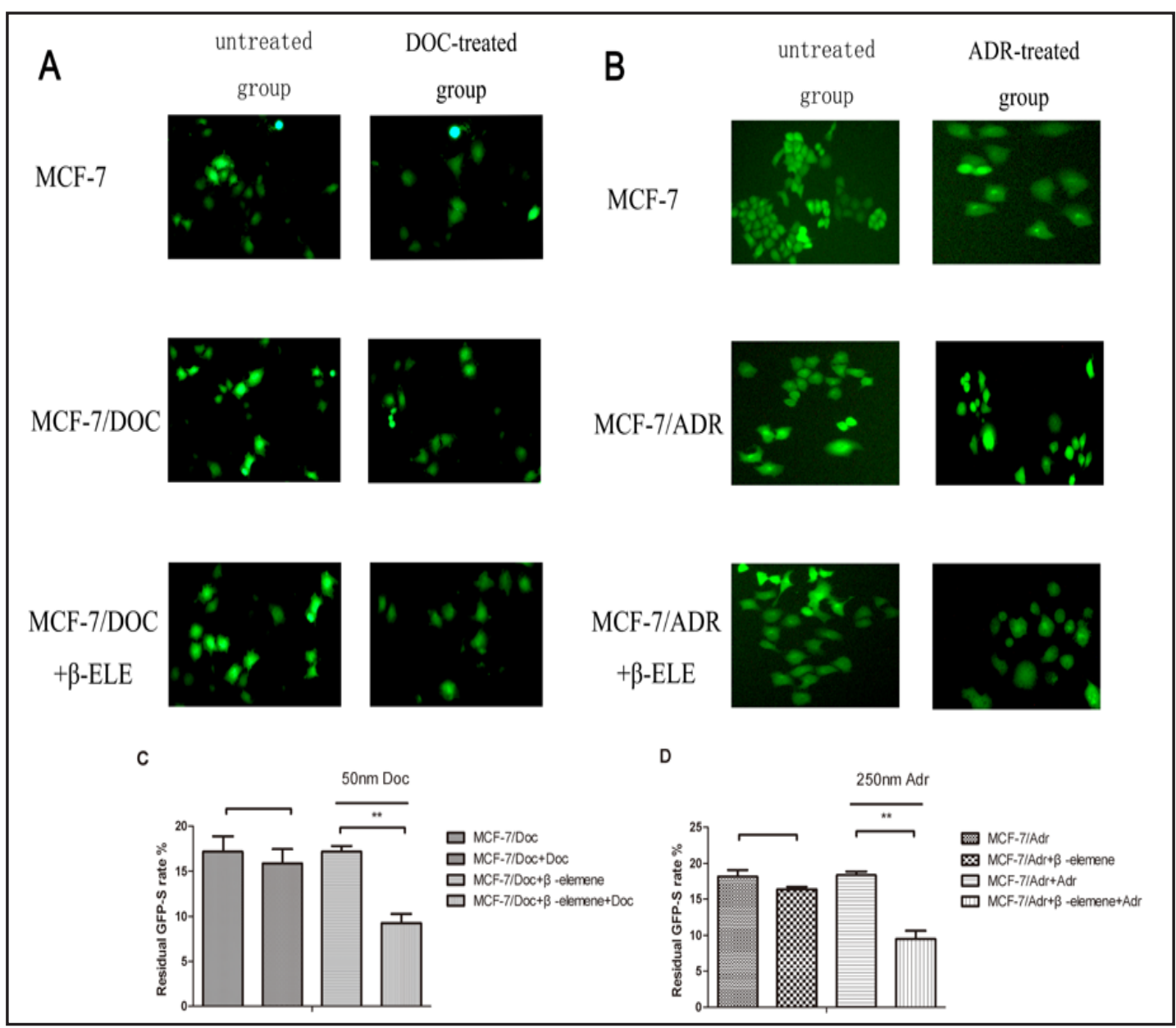

Fig. 4. The green fluorescent cells expression by dual luciferase activity assay in MCF-7 cells, MCF-7/Doc cells, MCF-7/Adr cells, MCF-7/Doc and MCF-7/Adr cells with $\beta$-elemene intervention (50 $\mu \mathrm{M} / \mathrm{L}$ ) for $30 \mathrm{~h}$ (Fig 4A, 4B). As shown in Fig 4C, 4D, between the untreated group and the Doc and Adr treated group, it shows clearly, after the MCF-7/Doc and MCF-7/Adr cells with $\beta$-elemene intervention, coped with the drug, the residual green fluorescent cells was significantly reduced more than MCF-7/Doc and MCF-7/Adr cells. Apoptotic rate of GFP-S was determined after cell mixture was treated with 50nm Doc or 250nm Adr for 30h. ${ }^{* *} \mathrm{P}<0.01, \mathrm{MCF}-7 /$ Adr or MCF-7/Doc vs MCF-7/Adr $+\beta$-elemene.

Fig. 5. The four miRNAs with consistent expression changes in MCF-7/Adr and MCF-7/Doc cells after $\beta$-elemene intervention $(50 \mu \mathrm{M} / \mathrm{L})$ for $30 \mathrm{~h}$. Compare with MCF-7/Adr and MCF-7/Doc without intervention, miR-29a, miR-222 and miR-452 levels were significantly lower, miR-34a levels were significantly higher, \#\#**P<0.01, MCF-7/Adr or MCF-7/Doc vs MCF-7/Adr $+\beta$-elemene.

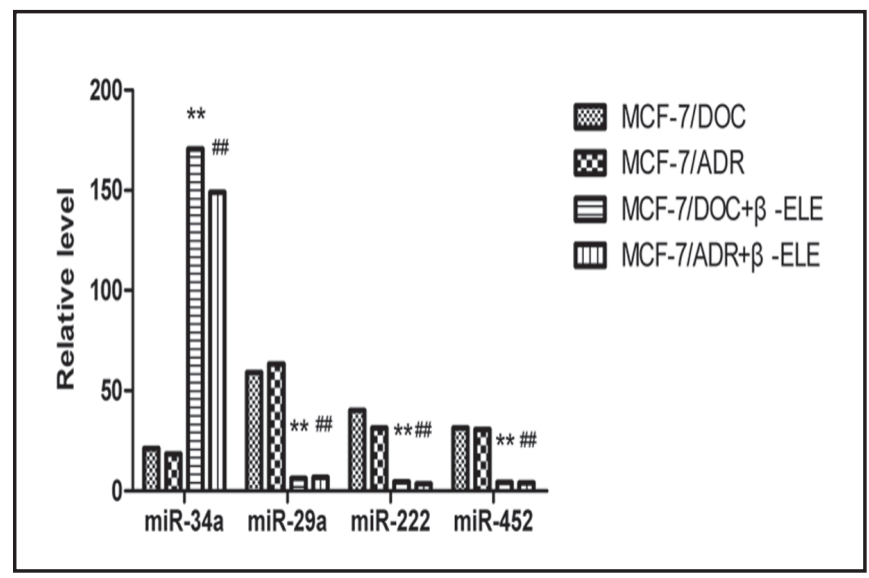

As shown in Fig. 6A, the PTEN expression in MCF-7/Doc and MCF-7/Adr cells was significantly decreased, compared with MCF-7 cells. However, the PTEN expressions in 


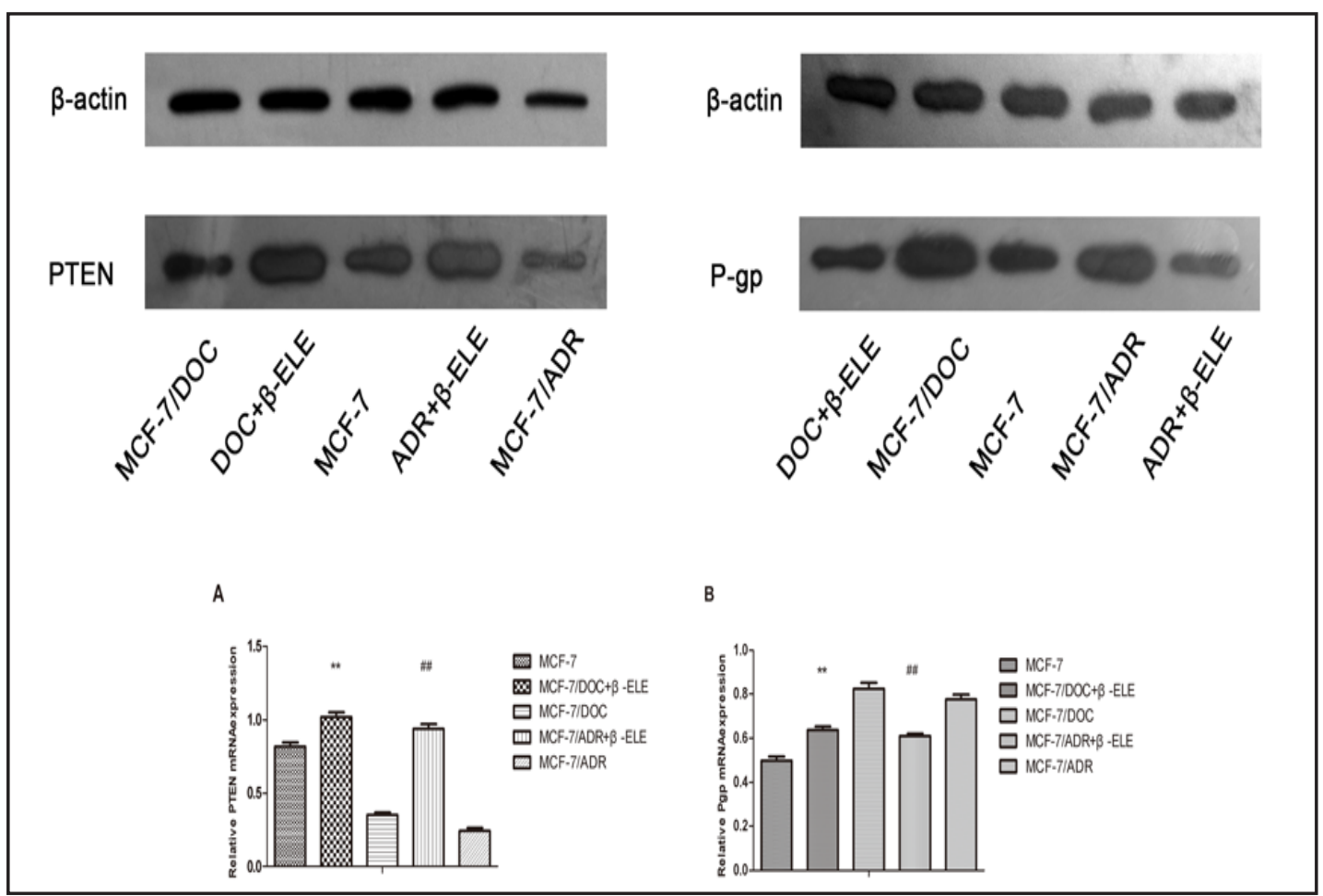

Fig. 6. The expression of PTEN mRNA, PTEN proteins levels (Fig. 6A) and Pgp mRNA, Pgp proteins levels (Fig. 6B) in MCF-7, MCF-7/ADR and MCF-7/DOC cells with $\beta$-elemene intervention ( $50 \mu \mathrm{M} / \mathrm{L}$ ) by Western blot. MCF-7/DOC and MCF-7/ADR cells treated with DMSO (control) or $\beta$-elemene for 30h, $\beta$-actin was used as a loading control in Western blot. A representative pattern is shown from three independent experi-

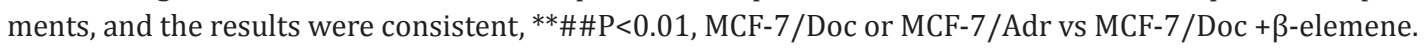

MCF-7/Doc treated with $\beta$-elemene $(50 \mu \mathrm{M} / \mathrm{L})$ and MCF-7/Adr cells treated with $\beta$-elemene $(50 \mu \mathrm{M} / \mathrm{L})$ were significantly increased when compared with the untreated ones. Also in Fig. 6B, the results showed that the Pgp expression in MCF-7/Doc and MCF-7/Adr cells was significantly increased, compared with MCF-7 cells; however, the Pgp Expression was significantly decreased in MCF-7/Doc with the intervention of $\beta$-elemene and MCF-7/Adr cells with the intervention of $\beta$-elemene compared with the untreated MCF-7/Doc and MCF7/Adr cells.

\section{Discussion}

Breast cancer is the most common cancer for women all over the world, Adr and Doc are two chemotherapeutic agents commonly used in the treatment of breast cancer, especially in recurrent or metastatic patients. One of the most important factors for the limited advances applied in cancer treatment is acquired drug resistance. In this study, we proved $\beta$-elemene could mediate the MDR specific miRNA, then regulate the corresponding target genes PTEN and Pgp -- reversing the drug-resistant of BCA cells.

Recently, the role of miRNAs in regulating drug resistance is reported. MiRNAs are a class of small non-coding RNAs with 18-25 nucleotides in length, which have been associated with every aspect of tumor biology, including acquisition of resistance to various chemotherapeutic agents.[5, 6] There are several mechanisms have recently been shown to be targeted by miRNAs in drug-resistant breast cancer, including: decreased intracellular drug concentrations; mediated by drug transporters and metabolic enzymes; impaired cellular responses that affect cell cycle arrest, apoptosis; DNA repair and alterations in the availability of drug targets [8]. 
We previously showed that $\beta$-elemene significantly suppresses breast cancer cells growth and proliferation $[15,16], \beta$-elemene has a wide range of applications in traditional medicine, it has been studied as an agent capable of reversing resistance to chemotherapy $[17,18]$. Previous Chinese publication has shown that by up-regulating the expression of $\mathrm{c}-\mathrm{Cbl}$ and $\mathrm{Cbl}-\mathrm{b}$, which leads to inhibition of PI3K/Akt signaling and down-regulation of Pgp expression [19]. $\beta$-elemene enhanced the sensitivity of A549/DDP cells to cisplatin and reversed the drug resistance of A549/DDP cells, it enhances susceptibility to cisplatin in resistant ovarian carcinoma cells via downregulation of ERCC- 1 and XIAP and inactivation of JNK. [20, 21]. This recent discovery shows that elemene-induced reversal of tamoxifen resistance in MCF-7 cells through oestrogen receptor $\alpha(E R \alpha)$ re-expression and it also shows that estrogen receptors can combine with the primary transcript of miRNAs to modify its biogenesis process [22].

In the present work, the efficacy of $\beta$-elemene in reversing the MDR of Doc and Adr cells was evaluated first via the MTT approach. The results demonstrated that $\beta$-elemene alone ranging from 10 to $30 \mu \mathrm{M} / \mathrm{L}$ did not display a significant anti-proliferative effect on Adr and Doc cells, while it at $50 \mu \mathrm{M} / \mathrm{L}$ enhanced the cytotoxicity toward the two cells. After the two cells were exposed to $50 \mu \mathrm{M} / \mathrm{L} \beta$-elemene for $30 \mathrm{~h}$, there was a pronounced increase in the apoptosis rate. Furthermore, it shows time dependence and concentration dependence. The MCF-7/Doc and MCF-7/Adr cells with $\beta$-elemene intervention coped with the drug was significantly reduced more than MCF-7/Doc and MCF-7/Adr cells; suggesting that $\beta$-elemene can reverse drug resistance by synergistic action.

Secondly, in order to verify another molecular pathway revolved on the impact of $\beta$-elemene in anticancer; we proceeded with miRNA expression profiling analysis, which aims to test the specific regulators of $\beta$-elemene-mediated anti-cancer properties in MCF-7 cells. From the miRNA expression profiles, we recognized differentially expressed miRNAs and consistent expression changes miRNAs in MCF-7/Adr and MCF-7/Doc cells with $\beta$-elemene intervention. This not only shows that resistant breast cancer cells with $\beta$-elemene intervention may have characteristics that can significantly change the miRNA expression, but also imply that the cells had mutual pathways along drug resistant specific pathways in selected MCF-7/Adr and MCF-7/Doc cells. In order to verify whether the differential miRNAs expression has a major influence in preventing the process of acquiring drug resistance with $\beta$-elemene intervention, we conducted related experiments. The results showed that miR-34, miR-222, miR-452 and miR-29a can changed the characteristics of drug resistant BCA cells to Doc and Adr. We therefore conclude that $\beta$-elemene could mediate MDR related miRNA expression to reduce the drug resistance of breast cancer. This study could contribute to understanding of the miRNAs roles in reversing drug resistance in BCA.

MDR of tumor cells is often associated with overexpression of Pgp and lower expression PTEN, finally leads to chemotherapeutic failure $[13,14]$. To explore a possible role of Pgp and PTEN in the effect of $\beta$-elemene on reversing drug resistance, we assessed PTEN and Pgp expression in beast cancer cells treated with $\beta$-elemene. Taken together, our results clearly indicate that $\beta$-elemene effectively sensitized drug resistant BCA cells to Doc and Adr through a signaling pathway involving regulation of PTEN and Pgp.

In summary, several studies have shown that $\beta$-elemene agent enhances sensitivity to chemotherapy in human breast cancer cell lines, it was approved as a national first-class new agent and phase II clinicaltrials are currently underway. We were able to establish that $\beta$-elemene not only causes a strong anticancer effect via activation of the apoptotic pathway to induce BCA cell apoptosis, but also the anticancer effect and reverse the drug resistance of $\beta$-elemene have a synergistic effect, $\beta$-elemene can effect target gene expression through transcriptional pathway; therefore, we conclude that miRNA plays a major influence in the $\beta$-elemene-mediated effect of MCF-7/Adr and MCF-7/Doc cells, particularly, the four specified drug resistant miRNAs. Moreover, this study provides a novel insight of the molecular mechanisms of $\beta$-elemene reversing tumor resistance by using the latest technology to detect miRNA communication mechanisms, The strategic in-depth exploration of MDR reducing expression mechanisms could help establish an alternative way of improving chemotherapy 
treatments. However, this study still has some shortcomings that need to be discussed. If animal models are embraced into our study -- through pharmaceutical interventions on the above subjects -- we could further explore how Chinese medicine can inverse and inhibit chemotherapy resistance in BCA from a clinical perspective; which could make our conclusions more persuasive.

\section{Acknowledgements}

This work was supported by grants from the National Natural Science Foundation of China (81272470).

\section{Disclosure Statement}

The authors declare no conflicts of interest.

\section{References}

1 Li QQ Wang G, Reed E, Huang L, Cuff CF: Evaluation of cisplatin in combination with $\beta$-elemene as a regimen for prostate cancer chemotherapy. Basic Clin Pharmacol Toxicol 2010;107:868-876.

2 Zhang X, Zhang Y, Li Y: $\beta$-elemene decreases cell invasion by upregulating E-cadherin expression in MCF-7 human breast cancer cells. Oncol Rep 2013;30:745-750.

-3 Xu HB, Li L, Fu J, Mao XP, Xu LZ: Reversion of multidrug resistance in a chemoresistant human breast cancer cell line by $\beta$-elemene. Pharmacology 2012;89:303-312.

-4 Guo HQ Zhang GN, Wang YJ, Zhang YK, Sodani K, Talele TT, Ashby CR Jr, Chen ZS: $\beta$-elemene, a compound derived from Rhizoma zedoariae, reverses multidrug resistance mediated by the ABCB1 transporter. Oncol Rep 2014;31:858-866.

5 Ratert N, Meyer HA, Jung M, Lioudmer P, Mollenkopf HJ, Wagner I, Miller K, Kilic E, Erbersdobler A, Weikert S, Jung K: MiRNA Profiling Identifies Candidate miRNAs for Bladder Cancer Diagnosis and Clinical Outcome. J Mol Diagn 2013;15:695-705.

6 Chen WX, Hu Q, Qiu MT, Zhong SL, Xu JJ, Tang JH, Zhao JH: MiR-221/222: promising biomarkers for breast cancer. Tumour Biol 2013;34:1361-1370.

7 Jia AY, Castillo-Martin M, Domingo-Domenech J, Bonal DM, Sánchez-Carbayo M, Silva JM, Cordon-Cardo C: A Common MicroRNA Signature Consisting of miR-133a, miR-139-3p, and miR-142-3p Clusters Bladder Carcinoma in Situ with Normal Umbrella Cells. Am J Pathol 2013;182:1171- 1179.

-8 Kutanzi KR, Yurchenko OV, Beland FA, Checkhun VF, Pogribny IP: MicroRNA-mediated drug resistance in breast cancer. Clin Epigenetics 2011;2:171-185.

-9 Jiao X, Zhao L, Ma M, Bai X, He M, Yan Y, Wang Y, Chen Q, Zhao X, Zhou M, Cui Z, Zheng Z, Wang E, Wei M: MiR-181a enhances drug sensitivity in mitoxantone-resistant breast cancer cells by targeting breast cancer resistance protein (BCRP/ABCG2). Res Treat Cancer Breast 2013;139:717-730.

$\rightarrow 10$ Zhong S, Li W, Chen Z, Xu J, Zhao J: MiR-222 and miR-29a contribute to the drug-resistance of breast cancer cells.Gene 2013;531:8-14.

11 Li XJ, Ji MH, Zhong SL, Zha QB, Xu JJ, Zhao JH, Tang JH: MicroRNA-34a modulates chemosensitivity of breast cancer cells to adriamycin by targeting Notch1. Arch Med Res 2012;43:514-521.

12 Zhang Y, Mu XD, Li EZ: The Role of E3 Ubiquitin Ligase Cbl Proteins in $\beta$-Elemene Reversing Multi-Drug Resistance of Human Gastric Adenocarcinoma Cells. Int J Mol Sci 2013;14: 0075-10089.

13 Yang L, Li N, Wang H, Jia X, Wang X, Luo J: Altered microRNA expression in cisplatin-resistant ovarian cancer cells and upregulation of miR-130a associated with MDR1/P-glycoprotein-mediated drugresistance. OncolRep 2012;28:592-600. 
14 Wang F, Li T, Zhang B, Li H, Wu Q Yang L, Nie Y, Wu K, Shi Y, Fan D: MicroRNA-19a/b regulates multidrug resistance in human gastric cancer cells by targeting PTEN. Biochem Biophys Res Commun 2013;434:688694.

15 Zhang X, Li Y, Zhang Y, Song J, Wang Q, Zheng L, Liu D: Beta-elemene blocks epithelial-mesenchymal transition in human breast cancer cell line MCF-7 through Smad3-mediated down-regulation of nuclear transcription factors. PLOS One 2013;8:e58719.

-16 Ding XF, Shen M, Xu LY: 13, 14-bis (cis-3, 5-dimethyl-1-piperazinyl)- $\beta$-elemene, a novel $\beta$-elemene derivative, shows potent antitumor activities via inhibition of mTOR in human breast cancer cells. Oncol Lett 2013;5:1554-1558.

17 Lu X, Wang Y, Luo H, Qiu W, Han H, Chen X, Yang L: $\beta$-elemene inhibits the proliferation of T24 bladder carcinoma cells through upregulation of the expression of Smad4. Mol Med Rep 2013;7:513-518.

18 Lee RX, Li QQ Reed E: $\beta$-elemene effectively suppresses the growth and survival of both platinum-sensitive and -resistant ovarian tumor cells. Anticancer Res 2012;32:3103-3113.

19 Zhang Y, Mu XD, Li EZ: The Role of E3 Ubiquitin Ligase Cbl Proteins in $\beta$-Elemene Reversing Multi-Drug Resistance of Human Gastric Adenocarcinoma Cells. Int J Mol Sci 2013;10:10075-10089.

20 Li QQ Lee RX, Liang H, Wang G, Li JM, Zhong Y, Reed E: $\beta$-elemene enhances susceptibility to cisplatin in resistant ovarian carcinoma cells via downregulation of ERCC-1 and XIAP and inactivation of JNK. Int J Oncol 2013;43:721-728.

21 Yao CC, Tu YR, Jiang J, Ye SF, Du HX, Zhang Y: $\beta$-elemene reverses the drug resistance of lung cancer A549/ DDP cells via the mitochondrial apoptosis pathway. Oncol Rep 2014;31:2131-2138.

22 Zhang B, Zhang X, Tang B, Zheng P, Zhang Y: Investigation of elemene-induced reversal of tamoxifen resistance in MCF-7 cells through oestrogen receptor $\alpha(E R \alpha)$ re-expression. Breast Cancer Res Treat 2012;136:399-406. 